\title{
Métodos de Recuperação Pós-exercício: uma Revisão Sistemática
}

\author{
Post-exercise Recovery Methods: a Systematic Review
}

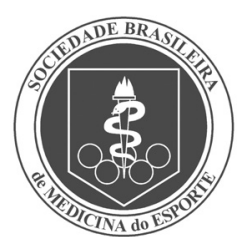

Artigo de Revisão
Carlos Marcelo Pastre?,

Fábio do Nascimento Bastos², Jayme Netto Júnior²,

Luiz Carlos Marques Vanderlei',

Rosangela Akemi Hoshi ${ }^{1}$

\section{Programa de Mestrado em} Fisioterapia. Faculdade de Ciências e Tecnologia FCT/Unesp. Presidente Prudente, SP.

2. Departamento de Fisioterapia. Faculdade de Ciências e Tecnologia FCT/Unesp. Presidente Prudente, SP.

\section{Endereço para correspondência:}

Faculdade de Ciências e Tecnologia - Unesp.

Departamento de Fisioterapia. Rua Roberto Simonsen, 305 19060-900 -Presidente Prudente, SP. E-mail: pastre@fct.unesp.br

Submetido em 22/02/2008 Versão final recebida em 28/06/2008 Aceito em 24/10/2008

\section{RESUMO}

A recuperação pós-exercício consiste em restaurar os sistemas do corpo a sua condição basal, proporcionando equilíbrio e prevenindo a instalação de lesões e, nesse sentido, torna-se aspecto importante de todo programa de condicionamento físico, em quaisquer níveis de desempenho, mas, sobretudo nos mais elevados. O objetivo desta revisão foi reunir informações e descrever as respostas proporcionadas por métodos recuperativos pós-exercício, como crioterapia, contraste, massagem e recuperação ativa, constituindo uma fonte de atualização do referido tema. Utilizaram-se os bancos de dados MedLine, Scielo e Lilacs, como lista de periódicos, o SportsDiscus. Foram incluídos no estudo somente ensaios clínicos randomizados controlados e não-controlados, além de artigos de revisão referentes ao tema proposto. Optou-se por procurar os termos: cryotherapy, massage, active recovery, thermotherapy, immersion e exercise, individualmente e em cruzamentos. Como achado, observou-se que alguns estudos relatam que a crioterapia é prejudicial em se tratando de recuperação pós-exercício, pois reduz o desempenho imediatamente após a aplicação da técnica. Por outro lado, estudos apontam como sendo benéfica, pois reduzem o nível de creatinaquinase após alta intensidade de esforço, evitando danos musculares. Para o contraste, embora apresente significância em se tratando de remoção de lactato sanguíneo, sua efetividade necessita ser mais bem discutida. Na massagem e na recuperação ativa, os principais vieses descritos dizem respeito à pressão exercida e à intensidade do exercício, respectivamente. Entre as técnicas, as que parecem ter efeitos semelhantes são o contraste e a recuperação ativa, no que tange à remoção de lactato e diminuição da creatinaquinase. Ressalta-se que o tempo de exposição é de fundamental importância para todos os métodos. Entretanto, diversos estudos não se propõem a identificar os reais efeitos fisiológicos promovidos pelas técnicas, utilizando-as de modo inipiente. Portanto, a inconsistência dos resultados encontrados sugere que a análise das variáveis utilizadas como método de recuperação deve ser mais bem controlada.

Palavras-chave: crioterapia, massagem, recuperação de função fisiológica, imersão.

\section{ABSTRACT}

The post-exercise recovery consists in restoring the body systems to baseline condition, providing balance and preventing injuries installation and, in that sense; it becomes an important aspect of every fitness program, at any levels of performance, but especially in higher levels. The objective of this review was to gather information and to describe the responses provided by post-exercise recovery methods, such as cryotherapy, contrast water immersion, massage and active recovery, providing an update on this issue. MedLine, Scielo and Lilacs databases were used, as well as the SportsDiscus list of journals. Only randomized controlled and non-controlled clinical essays, in addition to review articles concerning the proposed topic were included. Our choice was for the search terms: cryotherapy, massage, active recovery, thermotherapy, immersion and exercise, individually and combined. It was observed that some studies report that cryotherapy is harmful concerning post-exercise recovery, once it reduces performance immediately after the technique application. On the other hand, studies point it as being beneficial due to its reduction in the creatine kinase level after exercise, avoiding hence muscle damage. Concerning contrast water immersion, although it presents significance when it comes to blood lactate removal, its effectiveness needs to be better discussed. Regarding massage and active recovery, the main described biases relate to the pressure and intensity of the exercise, respectively. Among the techniques, contrast water immersion and active recovery seem to have similar effects concerning lactate removal and creatine kinase decrease. It is highlighted that the exposure time is crucial for all methods. However, several studies do not try to identify the real physiological effects promoted by the techniques, having them in limited use. Therefore, the inconsistency of the results found suggests that the assessed variables used as a recovery method should be better controlled.

Keywords: cryotherapy, massage, recovery of physiological function, immersion. 


\section{INTRODUÇÃO}

O sucesso dos processos de melhora do desempenho e prevenção de lesões depende da qualidade da transição entre os estímulos do treinamento físico, além da sistematização da prescrição do exercício. Nesse sentido, uma adequada recuperação torna-se um aspecto importante de todo programa de condicionamento, tanto para atletas, como técnicos e diversos profissionais ligados à área da saúde ${ }^{(1-3)}$. A recuperação pós-exercício consiste em restaurar os sistemas do corpo a sua condição basal, determinando a homeostase $e^{(4)}$.

A negligência ao tempo necessário para restauração de substratos utilizados durante o esforço antes de submeterem-se a um novo estímulo caracteriza uma condição inadequada, pois impedem que o organismo se mantenha em estado ótimo para realização da prática atlética, limitando o desempenho e aumentando os riscos de lesões ${ }^{(3,5-9)}$. Para potencializar a recuperação, tem-se observado, na prática, a utilização de vários métodos, como massagem ${ }^{(9-11)}$, exercícios ativos $^{(9,12)}$, contraste ${ }^{(13,14)}$ e crioterapia ${ }^{(1,15-17)}$.

Em se tratando de métodos recuperativos após alta intensidade de esforço, observa-se a realização de exercícios ativos, sendo estes considerados um trabalho contínuo aeróbio e de baixa intensidade ${ }^{(0,13)}$. Estudos ${ }^{(11,18-23)}$ apontam como intensidade ótima de esforço o intervalo entre 20 e $50 \%$ do $\mathrm{VO}_{2 \text { máx }}$ para que ocorra eficiente remoção de lactato sanguíneo, seja por oxidação ou conversão em glicose ou aminoácidos. Tal processo é amplamente descrito na literatura, conforme sugerem os estudos de revisão de Barnett ${ }^{(3)}$ e Tomlin e Wenger ${ }^{(4)}$.

A técnica de contraste consiste na alternância de exposição ao frio e calor, com o intuito de aumentar o metabolismo e, no esporte, tem sido utilizada nos processos de recuperação visando, também, à remoção do lactato sanguíneo ${ }^{(13)}$. Ainda no âmbito da termoterapia, observa-se com frequência a utilização da crioterapia, que consiste na redução da temperatura tecidual por condução; promove respostas relacionadas ao sistema de termorregulação do corpo, podendo tanto aumentar como diminuir o metabolismo ${ }^{(2)}$. Morton ${ }^{(14)}$ e Wilcock et al.(2) descrevem que tais recursos podem promover maior percepção subjetiva de recuperação.

Outra técnica utilizada amplamente no meio esportivo é a massagem, definida como manipulação mecânica dos tecidos do corpo com movimentos rítmicos e cadenciados. Os objetivos são a redução da dor e edema e a aceleração da remoção de lactato, pelo aumento de fluxo sanguíneo ${ }^{(3,24)}$.

Alguns resultados têm sido observados a partir dos modelos de recuperação descritos, seja com contraste ${ }^{(13,14)}$, crioterapia $^{(6,15,17)}$ ou massagem ${ }^{(24)}$, além da própria recuperação ativa ${ }^{(25,26)}$. Contudo, a falta de padronização para utilização das técnicas e controle das variáveis determina dificuldades para comparação de resultados entre estudos de mesma natureza. Nesse sentido, atenta-se para necessidade de melhor definição de parâmetros relacionados ao gerenciamento de cada técnica, como tempo de exposição, temperatura e intensidade de aplicação.

Portanto, o objetivo desta revisão foi reunir informações e descrever as respostas proporcionadas por métodos recuperativos pós-exercício, como crioterapia, contraste, massagem e recuperação ativa, constituindo uma fonte de atualização do referido tema.

\section{METODOLOGIA}

Os artigos potencialmente úteis foram obtidos por meio de pesquisa bibliográfica nos bancos de dados online Medline (Medical Literature, Analysis and Retrieval System Online), Scielo (Scientific Electronic Library Online) e Lilacs (Literatura Latino-americana e do Caribe de Informação em Ciências da Saúde), e, como lista de periódicos, o Sportdiscus.
Optou-se por procurar os termos cryotherapy, massage, active recovery, thermotherapy, immersion e exercise, individualmente e em cruzamentos. Nas bases de dados Lilacs e Scielo também foram utilizados os termos em português: crioterapia, massagem, recuperação de função fisiológica, imersão e exercício.

A partir dos cruzamentos observou-se a ocorrência de 237 artigos no total. Incluíram-se apenas os que se referiam à recuperação pós-exercício por meio das técnicas abordadas neste estudo, selecionando-se 46 artigos para integrar a revisão. Foram excluídos artigos que, mesmo apresentando os unitermos utilizados para busca, não contemplavam a relação entre técnica e recuperação. Assim, os 191 títulos excluídos apresentavam conteúdos diversos, relativos à terapêutica, prevenção e desempenho.

O estudo de revisão foi restrito ao período de janeiro de 2000 até dezembro de 2007 para os estudos relacionados à recuperação ativa, e de janeiro de 2004 até dezembro de 2007 para os referentes ao contraste e massagem; para o termo crioterapia, a revisão foi restrita a janeiro de 1984 até dezembro de 2007.

Todos os artigos obtidos na busca eletrônica tiveram seus resumos extraídos e analisados de maneira independente. Objetivando selecionar os estudos de maior evidência científica, contemplamos os ensaios clínicos randomizados controlados e não-controlados baseados em técnicas recuperativas, mais especificamente, crioterapia, contraste, massagem e recuperação ativa. Optou-se por não restringir a condição clínica da amostra de cada estudo devido ao limitado referencial bibliográfico referente ao tema proposto. Selecionaram-se, também, estudos de revisão que apresentassem como tema métodos de recuperação pós-exercício. Por fim, no total compuseram esta revisão 36 estudos referentes a ensaios clínicos randomizados, sendo 34 controlados e dois não-controlados, além de 10 artigos de revisão.

A partir da obtenção e leitura dos artigos, suas referências bibliográficas foram rastreadas à procura de outros artigos potencialmente utilizáveis. Esse trabalho repetiu-se tantas vezes quanto necessário, até haver a convicção de que nenhuma das referências obtidas continha estudos que já não tivessem sido identificados. Não houve restrição quanto à língua nesta pesquisa.

\section{APRESENTAÇÃO E DISCUSSÃO DOS RESULTADOS}

\section{Crioterapia}

Diversos estudos utilizaram a crioterapia com o objetivo de recuperação pós-exercício ${ }^{(1,15-17,27)}$. Desse modo, aparecem protocolos dos mais variados ${ }^{(2)}$.

Paddon-Jones e Quigley(15) utilizaram cinco imersões em água e gelo com temperatura de $5 \pm 1^{\circ} \mathrm{C}$, por 20 minutos, imediatamente após o exercício e, as seguintes, de uma em uma hora após o teste. Ohnishi et al. ${ }^{(6)}$, com semelhante protocolo em relação ao tempo de exposição à técnica, utilizaram imersão, uma única vez, à temperatura de $10 \pm 1{ }^{\circ} \mathrm{C}$. Os resultados mostraram que não houve significância estatística entre os grupos experimentais e controle em ambos os estudos ${ }^{(6-15)}$.

Armstrong et al.(28) utilizaram média de 16 minutos de imersão em água e gelo com temperatura entre $1^{\circ} \mathrm{C}$ e $3^{\circ} \mathrm{C}$. Eston e Peters ${ }^{(16)} \mathrm{e}$ Yanagisawa et al.(8) utilizaram a mesma técnica por 15 minutos, com temperatura de $15 \pm 1^{\circ} \mathrm{C}$ e $5^{\circ} \mathrm{C}$, respectivamente. Para Armstrong et al.(28) , a imersão foi benéfica para tirar o sujeito do estado de hipertermia, além de melhorar a sensação de recuperação pós-exercício. Eston e Peters ${ }^{(16)}$ e Yanagisawa et al..$^{(8)}$ obtiveram como resultado diminuição da CK no terceiro e no quarto dia, respectivamente, após o esforço.

Outros autores utilizaram a técnica de imersão por tempos menores, como 12 minutos ${ }^{(29)}, 10$ minutos $^{(17)}$, cinco minutos ${ }^{(30)}$ e até um minuto(1). 
Além desses métodos, Isabell et al. ${ }^{(31)}$, Yackzan et al. ${ }^{(32)}$ e Howatson e van Someren ${ }^{(33)}$ utilizaram a massagem com gelo por 15 minutos. E obtiveram resultados satisfatórios, como melhor amplitude de movimento imediatamente pós-exercício ${ }^{(26)}$, bem como diminuição de $\mathrm{CK}^{(33)}$. Para Isabell et al.(31), não houve significância estatística para as variáveis estudadas.

Tais fatos estão intimamente relacionados com os efeitos fisiológicos da crioterapia, que incluem diminuição da frequência cardíaca e débito cardíaco, aumento da pressão arterial e resistência periférica. 0 aumento da resistência periférica é devido ao sangue ser redirecionado para a periferia, de modo a manter a temperatura corpórea. O consumo de oxigênio e o metabolismo também aumentam para auxiliar na manutenção da temperatura ${ }^{(2)}$.

Além desses efeitos, é importante notar que a crioterapia reduz a permeabilidade celular de vasos sanguíneos, linfáticos e capilares devido à vasoconstrição, fazendo com que ocorra diminuição da difusão dos fluidos nos espaços intersticiais. Essa cascata de respostas é favorável à diminuição da inflamação provocada por danos teciduais, além de reduzir a dor, o edema e o espasmo muscular ${ }^{(1,2,16)}$.

Ainda no âmbito fisiológico, nota-se que componentes neurais também são afetados com baixas temperaturas. O resfriamento dos tecidos diminui a transmissão nervosa, reduzindo a liberação de acetilcolina e, possivelmente, estimulando células superficiais inibitórias a aumentar o limiar de dor ${ }^{(2)}$. A partir disso, Wilcock et al. ${ }^{(2)}$ supõem dois efeitos para transmissão do impulso nervoso com a utilização da crioterapia: (i) reduzir o nível de percepção de dor (analgesia); e (ii) reduzir o espasmo muscular.

Pöyhönem e Avela ${ }^{(34)}$ concluíram que baixas temperaturas reduzem a sensação de fadiga muscular, podendo ser esse um efeito psicológico. Outra hipótese é que a imersão pode modificar respostas em nível periférico e central; dessa forma, a redução na sensação de fadiga pode estar relacionada com diminuição da resposta neuromuscular.

Em síntese, alguns autores acreditam que, após exercício de alta intensidade de esforço, a crioterapia seja imprópria para redução de marcadores biológicos, tais quais lactato sanguíneo ${ }^{(8,35)}, \mathrm{CK}^{(17,31)}$, interleucina-6(6) e mioglobina ${ }^{(36)}$, para redução de sinais e sintomas como percepção do músculo dolorido e circunferência do membro, ou, ainda, para o restabelecimento pleno de funções como amplitude de movimento e torque isométrico máximo ${ }^{(36)}$. Todavia, outros estudos ${ }^{(8,16,22)}$ apontam a capacidade de redução da concentração de CK e de lactato sanguíneo após esforço intenso.

A diferença entre os estudos supracitados pode ter ocorrido devido às metodologias utilizadas, que diferem em relação ao controle das variáveis. Nesse sentido, deve-se atentar à temperatura da água utilizada no experimento, modelo de esforço e tempo de exposição à técnica, para que se consiga uma comparação efetiva e possibilite levantar hipóteses mais concretas sobre as incongruências encontradas.

\section{Contraste}

Dentre as técnicas utilizadas para recuperação pós-exercício, o contraste, apesar da maneira incipiente, também tem sido estudado. Tal técnica consiste em alternar a temperatura de imersão em quente e frio ou vice-versa da estrutura a ser tratada(13,14).

Coffey et al. ${ }^{(18)}$, em estudo com 14 sujeitos fisicamente ativos, concluíram que o contraste utilizado por 15 minutos demonstra melhor sensação de recuperação quando comparado com o grupo recuperação ativa, embora a remoção do lactato sanguíneo não tivesse significância estatística entre esses dois grupos.

Estudos recentes ${ }^{(14,37)}$ também mostram a efetividade da técnica, ratificando a informação de que o contraste acelera a remoção de catabólitos produzidos durante alta intensidade de esforço. Além disso, descrevem o relaxamento da musculatura esquelética e meIhora da percepção subjetiva de recuperação com a aplicação da técnica.

A partir dos achados, alguns autores ${ }^{(2,13)}$ puderam concluir que a acelerada remoção do acido lático se deve ao efeito de vasoconstrição e vasodilatação promovido pela técnica. Além desses efeitos, Wilcock et al. ${ }^{(2)}$ e Morton ${ }^{(14)}$ supõem que a pressão hidrostática da água pode influir na remoção desses catabólitos. É importante ressaltar que, quanto mais rápida a eliminação do lactato sanguíneo, mais eficaz a recuperação ${ }^{(25)}$.

Devido ao número reduzido de estudos, não é adequado afirmar sua efetividade como ferramenta de recuperação. Cochrane ${ }^{(13)}$, em revisão, relata que a técnica em relação ao tratamento de lesões está bem fundamentada, mas quando se trata de acelerar o processo de recuperação, não se conhecem os verdadeiros efeitos fisiológicos. Portanto, faz-se necessário atentar para os aspectos metodológicos, como número de repetições (quente/frio), tempo total da técnica e variação de temperatura da água.

\section{Massagem}

Muitos profissionais ligados à área da medicina do esporte, baseados em observações e experiência prática, acreditam que a massagem pode apresentar efeitos benéficos, em se tratando de recuperação pós-exercício. Com isso, vários estudos mostram que a técnica, apesar de muito utilizada, não tem suas reais potencialidades definidas. Isso se deve à grande variedade de protocolos utilizados ${ }^{(24,38-40)}$.

Tais protocolos são referentes à população de estudo, tipo, duração e pressão da técnica aplicada. Zainuddin et al.(41), em estudo com 10 sujeitos, aplicaram massagem por 10 minutos imediatamente após 60 repetições de flexão do cotovelo e observaram redução no pico da concentração de CK, quando comparada com a do grupo controle. A massagem consistiu de deslizamentos, amassamentos e fricções por todo o membro superior.

Em estudo recente, Hart et al. ${ }^{(10)}$ utilizaram deslizamentos e amassamentos por cinco minutos com o objetivo de reduzir a água intramuscular e a sensação de dor após exercício excêntrico; os resultados mostraram que não há significância entre o grupo testado e controle. Em contradição, Mori et al.(42) aplicaram o mesmo tempo de massagem a fim de investigar os reais efeitos do fluxo sanguíneo na pele e no músculo, além da sensação de fadiga após exercício isométrico. Como resultado, destacaram que a circulação sanguínea aumenta na região tratada e que a sensação de fadiga muscular é menor quando comparada com a do grupo controle.

Segundo Weerapong et al.(24), a massagem alivia a dor muscular tardia, devido ao aumento do fluxo sanguíneo e do fluxo linfático, diminuindo a água intramuscular e a sensação de dor. Com isso, acelera a remoção de catabólitos, consequentemente, reduz o tempo de recuperação ${ }^{(39,42)}$.

Embora alguns estudos ${ }^{(39,42)}$ mostrem que a massagem aumenta o fluxo sanguíneo, Robertson et al. ${ }^{(40)}$ concluíram que não há aumento da circulação na massagem por 20 minutos, pelo fato de não haver diminuição do lactato sanguíneo após alta intensidade de esforço.

Contudo, apesar de a massagem ser utilizada com o intuito de reduzir o tempo de recuperação do atleta, melhorando seu desempenho(10) e até de prevenir lesões relacionadas ao esforço(24), seus resultados ainda não são claros, devido, principalmente, à utilização de diversos protocolos. Um dos vieses para análise da resposta das técnicas e comparação entre as mesmas diz respeito à pressão exercida sobre a estrutura a ser analisada. 
Tabela 1. Síntese das pesquisas relacionando crioterapia e recuperação pós-exercício.

\begin{tabular}{|c|c|c|c|c|c|}
\hline Autor & População de estudo & $\begin{array}{l}\text { Modelo de estresse e nível } \\
\text { de controle }\end{array}$ & $\begin{array}{l}\text { Modelo de recuperação e } \\
\text { controle }\end{array}$ & Variáveis estudadas & Resultados \\
\hline Armstrong et al. ${ }^{(28)}$ & $\begin{array}{l}21 \text { atletas de resistência, } \\
\text { ambos os sexos } \\
\text { ( } 35 \pm 3 \text { anos). }\end{array}$ & $11,5 \mathrm{~km}$ de corrida. & $\begin{array}{l}\text { Média de } 16^{\prime} \text { de imersão em } \\
\text { água e gelo }\left(1-3^{\circ} \mathrm{C}\right) \text {; e com } \\
\text { média de } 14^{\prime} \text { em temperatura } \\
\text { ambiente a } 24,4^{\circ} \mathrm{C} \text {. }\end{array}$ & $\begin{array}{l}\text { Temperatura retal, pressão ar- } \\
\text { terial (PA), frequência cardíaca } \\
(\mathrm{FC}) \text {, acuidade mental. }\end{array}$ & $\begin{array}{l}\text { A imersão mostrou-se benéfica para reduzir ou reverter o estado } \\
\text { de hipertermia. }\end{array}$ \\
\hline Douris et al. ${ }^{(30)}$ & $\begin{array}{l}16 \text { sujeitos do sexo } \\
\text { masculino (32 } \pm 6,3 \text { anos). }\end{array}$ & $\begin{array}{l}3 \text { contraçōes isométricas máxi- } \\
\text { mas por } 3^{\prime \prime} \text { com } 30^{\prime \prime} \text { de repouso } \\
\text { entre as séries em dinamôme- } \\
\text { tro isocinético. Membros supe- } \\
\text { riores. }\end{array}$ & $\begin{array}{l}\text { Imersão em água e gelo } \\
\left(10 \pm 1^{\circ} \mathrm{C}\right) \text { nos tempos } 5,10,15 \\
\text { e } 20^{\prime} \text { pós-exercício. }\end{array}$ & $\begin{array}{l}\text { Mensuração da força após a } \\
\text { técnica. }\end{array}$ & $\begin{array}{l}\text { 20' de imersão apresentou maior força quando comparado com } \\
\text { 5'. A força mensurada imediatamente pós- imersão de } 10^{\prime} \text { foi } \\
\text { maior quando comparada com a imersão de } 5 \text { e } 20^{\prime} \text {. }\end{array}$ \\
\hline Eston e Peters. ${ }^{(16)}$ & $\begin{array}{l}15 \text { sujeitos do sexo } \\
\text { feminino (22 } \pm 2 \text { anos). }\end{array}$ & $\begin{array}{l}8 \text { séries de } 5 \text { repetições con- } \\
\text { cêntricas máximas em dinamô- } \\
\text { metro isocinético. Intervalo de } \\
60 " \text { entre cada série. Membros } \\
\text { superiores. }\end{array}$ & $\begin{array}{l}\text { Imersão em água e gelo } \\
\left(15 \pm 1^{\circ} \mathrm{C}\right) \text { por } 15^{\prime}, \text { imediatamen- } \\
\text { te após exercício e a cada } 12 \mathrm{~h}, \\
\text { por } 3 \text { dias e grupo controle. }\end{array}$ & $\begin{array}{l}\text { CK, força isométrica, ângulo } \\
\text { do cotovelo (relaxado), dolo- } \\
\text { rimento muscular e circunfe- } \\
\text { rência do membro. }\end{array}$ & $\begin{array}{l}\text { O grupo controle apresentou menor ângulo de "relaxamento" } \\
\text { em relação ao grupo da imersão. No segundo e terceiro dia } \\
\text { a CK esteve aumentada no controle em relação à imersão. A } \\
\text { força isométrica esteve aumentada na imersaao em relação ao } \\
\text { controle, principalmente no terceiro dia. }\end{array}$ \\
\hline Hornery et al. ${ }^{(35)}$ & $\begin{array}{l}14 \text { ciclistas, ambos os } \\
\text { sexos }(29,85 \pm 5,18 \text { anos). }\end{array}$ & $\begin{array}{l}30^{\prime} \text { pedalando a } 75 \% \mathrm{VO}_{2 \text { máxo }} \\
\text { seguido de uma técnica recu- } \\
\text { perativa por } 10^{\prime} \text { e logo após, } \\
\text { pedalar por } 20^{\prime} \text { seguido de } 10^{\prime} \\
\text { de máximo trabalho. }\end{array}$ & $\begin{array}{l}\text { Recuperação por crioterapia } \\
\text { "jaqueta fria" por } 10^{\prime} \text { e grupo } \\
\text { controle. }\end{array}$ & $\begin{array}{l}\text { FC, concentração de lactato } \\
\text { [Lac], temperatura retal e per- } \\
\text { cep̧̧ão de esforço (Borg). }\end{array}$ & $\begin{array}{l}\text { O desempenho após } 10^{\prime} \text { de máxima intensidade de esforço } \\
\text { melhorou com a crioterapia. A FC aumentou no } 5^{\circ} \text { min. de } \\
\text { esforço máximo no grupo de crioterapia, bem como a [Lac] } \\
\text { aumentou no } 6^{\circ} \text { min após esforço. A percepção de esforço foi } \\
\text { maior no grupo controle no } 20^{\circ} \text { min, imediatamente após o } \\
\text { esforço sub-máximo. }\end{array}$ \\
\hline Howatson et al. ${ }^{(33)}$ & 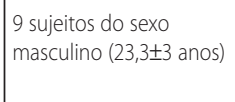 & $\begin{array}{l}3 \text { séries de } 10 \text { repetições de } \\
\text { flexão do cotovelo com } 70 \% \\
\text { de } 1 \text { RM }\end{array}$ & $\begin{array}{l}\text { Massagem com gelo e grupo } \\
\text { controle, imediatamente pós- } \\
\text { exerćíci, } 24 \text { e } 48 \mathrm{~h} .\end{array}$ & $\begin{array}{l}\text { CK, dor muscular tardia, circun- } \\
\text { ferência do membro e ampli- } \\
\text { tude de movimento. }\end{array}$ & $\begin{array}{l}\text { Após } 72 \mathrm{~h} \text { a CK mostrou-se diminuída no grupo massagem } \\
\text { com gelo. }\end{array}$ \\
\hline Howatson et al. ${ }^{(36)}$ & $\begin{array}{l}12 \text { sujeitos fisicamente } \\
\text { ativos, sexo masculino } \\
\text { (24,8 } \pm 5,3 \text { anos). }\end{array}$ & $\begin{array}{l}3 \text { séries de } 10 \text { repetições de } \\
\text { flexão do cotovelo usando um } \\
\text { dinamômetro isocinético e inter- } \\
\text { valo de } 3^{\prime} \text { entre as séries. }\end{array}$ & $\begin{array}{l}\text { Massagem com gelo por um } \\
\text { período de } 15^{\prime} \text { e o grupo pla- } \\
\text { cebo (ultrassom desligado) } \\
\text { por 5!. }\end{array}$ & $\begin{array}{l}\text { Percepção de músculo dolori- } \\
\text { do, circunferência do membro, } \\
\text { amplitude de movimento, tor- } \\
\text { que isométrico máximo, CKe a } \\
\text { mioglobina. }\end{array}$ & $\begin{array}{l}\text { A CK no grupo placebo continuou a aumentar após as primeiras } \\
96 \mathrm{~h} \text { pós-exercício; o grupo de massagem com gelo apresentou } \\
\text { pico nas primeiras } 48 \mathrm{~h} \text { pós-exercício e tendeu a decrescer. }\end{array}$ \\
\hline Isabell et al.(31) & $\begin{array}{l}22 \text { sujeitos, ambos os } \\
\text { sexos (20,3 } \pm 2,1 \text { anos). }\end{array}$ & $\begin{array}{l}30 \text { contrações excêntricas e con- } \\
\text { cêntricas dos músculos flexores } \\
\text { do cotovelo com carga de } 90 \% \\
\text { de 10RM. }\end{array}$ & $\begin{array}{l}\text { Massagem com gelo, mas- } \\
\text { sagem com gelo e exercício, } \\
\text { exercício e controle. Todos os } \\
\text { procedimentos foram de 15! }\end{array}$ & $\begin{array}{l}\text { Escala de dor, amplitude de } \\
\text { movimento, força e CK. }\end{array}$ & $\begin{array}{l}\text { Não houve significância entre as variáveis e os grupos estu- } \\
\text { dados. }\end{array}$ \\
\hline Ohnishi et al.(6) & $\begin{array}{l}16 \text { sujeitos do } \\
\text { sexo masculino } \\
(20,7 \pm 2,3 \text { anos). }\end{array}$ & $\begin{array}{l}3 \text { séries de } 8 \text { repetições num } \\
\text { ergômetro } 3 \text { vezes na semana } \\
\text { durante } 6 \text { semanas. Membros } \\
\text { superiores }\end{array}$ & $\begin{array}{l}\text { Imersão em gelo }\left(10 \pm 1^{\circ} \mathrm{C}\right) \text { e } \\
\text { grupo controle, ambas por } 20 \\
\text { minutos. }\end{array}$ & $\begin{array}{l}\text { Circunferência do membro, } \\
\text { força máxima, resistência } \\
\text { muscular, diâmetro da artéria } \\
\text { braquial, concentração de IL-6 } \\
\text { e VEGF. }\end{array}$ & $\begin{array}{l}\text { O diâmetro da artéria aumentou no grupo controle quando } \\
\text { comparado com a imersão. }\end{array}$ \\
\hline $\begin{array}{l}\text { Paddon-Jones } \\
\text { et al. }{ }^{(15)}\end{array}$ & 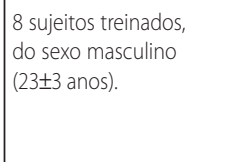 & $\begin{array}{l}8 \text { séries de } 8 \text { repetiçōes máximas } \\
\text { excêntricas, com carga corres- } \\
\text { pondente a } 110 \% \text { de } 1 \mathrm{RM} \text { com } \\
30 " \text { de repouso entre as séries. } \\
\text { Membros superiores. }\end{array}$ & $\begin{array}{l}\text { Cinco imersöes em gelo } \\
\left(5 \pm 1^{\circ} \mathrm{C}\right) \text { por } 20^{\prime} \text {, imediatamente } \\
\text { após o exercíco e as seguintes } \\
\text { de uma em uma hora e grupo } \\
\text { controle. }\end{array}$ & $\begin{array}{l}\text { Torque isométrico, desconfor- } \\
\text { to muscular, volume do braço, } \\
\text { força. }\end{array}$ & $\begin{array}{l}\text { Não houve significância entre os grupos em relação às variáveis } \\
\text { estudadas. }\end{array}$ \\
\hline Sellwood et al.11) & $\begin{array}{l}40 \text { sujeitos não treinados } \\
\text { ( } 21 \pm 3,1 \text { anos - grupo } \\
\text { controle, e } 21,4 \pm 4,3 \text { anos } \\
\text { grupo estudado). }\end{array}$ & $\begin{array}{l}5 \text { séries de } 10 \text { contrações máxi- } \\
\text { mas de extensão de joelho, com } \\
1 \text { ' de repouso entre as séries. } \\
120 \% \text { de } 1 \text { RM. }\end{array}$ & $\begin{array}{l}\text { Imersão em água e gelo } \\
\left(5 \pm 1^{\circ} \mathrm{C} \text { ) e grupo controle }\right. \\
\text { - imersão a temperatura de } \\
24^{\circ} \mathrm{C} \text {. Os sujeitos permaneciam } \\
\text { imersos por } 1^{\prime} \text { e fora do tambor } \\
1^{\prime} \text {, repetindo } 3 \text { ciclos. }\end{array}$ & $\begin{array}{l}\text { Escala de dor (EVA) para sentar } \\
\text { e ficar em repouso, alonga- } \\
\text { mento passivo, saltar, correr e } \\
\text { máxima contração isométrica, } \\
\text { dolorimento muscular, circun- } \\
\text { ferência do membro e CK. }\end{array}$ & $\begin{array}{l}\text { O grupo de imersão apresentou aumento da dor (sentar e fi- } \\
\text { car em repouso) após } 24 \mathrm{~h} \text { quando comparado com o grupo } \\
\text { controle. }\end{array}$ \\
\hline Yackzan et al. ${ }^{(32)}$ & $\begin{array}{l}20 \text { sujeitos do sexo } \\
\text { feminino (entre } 20 \text { e } \\
36 \text { anos). }\end{array}$ & $\begin{array}{l}\text { Contração excêntrica dos mús- } \\
\text { culos flexores do cotovelo, até } \\
\text { a exaustão. }\end{array}$ & $\begin{array}{l}\text { Massagem com gelo por } 15^{\prime} \text { e } \\
\text { grupo controle. Mediu-se nos } \\
\text { tempos, imediatamente, } 24 \text { e } \\
48 \mathrm{~h} \text { após o exercício, grupos } \\
\text { A,B e C, respectivamente. }\end{array}$ & $\begin{array}{l}\text { Escala de dor e amplitude de } \\
\text { movimento. }\end{array}$ & $\begin{array}{l}\text { Os sujeitos do grupo B sentiram melhora da dor quando com- } \\
\text { parados com o grupo controle e com o grupo C. Os sujeitos } \\
\text { do grupo A tiveram melhor amplitude de movimento quando } \\
\text { comparados com os do grupo B, que também tiveram ampli- } \\
\text { tude inferior à dos do grupo C. }\end{array}$ \\
\hline Yanagisawa et al ${ }^{(8)}$ & 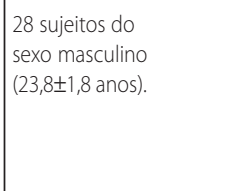 & \begin{tabular}{|l|} 
Flexão plantar em posição ortos- \\
tática, 5 séries de 20 repetiçães a \\
$30 \%$ da contração máxima, com \\
1'de repouso entre as séries.
\end{tabular} & $\begin{array}{l}\text { Crioterapia }\left(5^{\circ} \mathrm{C}\right) \text { por } 15^{\prime} \text { ime- } \\
\text { diatamente após o exercício, } \\
\text { imediatamente e } 24 \text { horas } \\
\text { após (dupla imersão) e grupo } \\
\text { controle. }\end{array}$ & $\begin{array}{l}\text { Ressonância magnética, ampli- } \\
\text { tude de movimento, CK, [Lac] } \\
\text { e escala de dor. }\end{array}$ & $\begin{array}{l}\text { O sóleo apresentou alteração nos 40' na dupla imersão, quando } \\
\text { comparado com os dois grupos. A amplitude foi menor no con- } \\
\text { trole quando comparado com os dois grupos. A [Lac] apresentou- } \\
\text { se em menor concentração na dupla imersão quando comparado } \\
\text { com a imersão. O grupo controle e a dupla imersão apresentaram } \\
\text { aumento da CK nas primeiras } 96 \text { horas após o exercício. }\end{array}$ \\
\hline Yeargin et al. ${ }^{(29)}$ & $\begin{array}{l}15 \text { sujeitos treinados } \\
\text { de ambos os sexos } \\
\text { (28 } \pm 2 \text { anos). }\end{array}$ & $\begin{array}{l}\text { Correr } 45^{\prime} \text { no solo, seguido de } \\
\text { uma pausa de 3', corria mais } 45^{\prime} \\
\text { e após 2-4' fazia-se uma inter- } \\
\text { venção por } 12 \text { '. Após } 15^{\prime} \text { inicia-se } \\
\text { uma corrida de } 2 \text { milhas. }\end{array}$ & $\begin{array}{l}\text { Imersão a } 5^{\circ} \mathrm{C} \text {, imersão a } 14^{\circ} \mathrm{C} \text { e } \\
\text { temperatura de } 29^{\circ} \mathrm{C} \text { em deter- } \\
\text { minado ambiente por } 12^{\prime} .\end{array}$ & $\begin{array}{l}\text { Temperatura retal, [Lac], FC, } \\
\text { sensaçãa térmica, teste de } \\
\text { urina, escala numérica visual e } \\
\text { tempo de corrida (2 milhas). }\end{array}$ & $\begin{array}{l}\text { Sensação térmica foi menor a } 5^{\circ} \mathrm{C} \text { e } 14^{\circ} \mathrm{C} \text { comparada com } 29^{\circ} \mathrm{C} \text {. } \\
\text { Temperatura retal no } 29^{\circ} \mathrm{C} \text { foi menor comparada com } 5^{\circ} \mathrm{C} \text { e } \\
14^{\circ} \mathrm{C} \text {, pós-corrida. Após a imersão a [Lac] foi menor no } 29^{\circ} \mathrm{C} \text { em } \\
\text { relação ao } 14^{\circ} \mathrm{C} \text {. A } 5^{\circ} \mathrm{C} \text { teve menor tempo de corrida em relação } \\
\text { ao } 29^{\circ} \mathrm{C} \text { com desempenho } 6 \% \text { melhor. }\end{array}$ \\
\hline Crowe et al. ${ }^{(27)}$ & $\begin{array}{l}13 \text { sujeitos de ambos os } \\
\text { sexos fisicamente ativos. }\end{array}$ & $\begin{array}{l}2 \text { tiros de aproximadamente } \\
30 " \text { num cicloergômetro. Com } 1 \\
\text { hora de intervalo entre os dois }\end{array}$ & $\begin{array}{l}\text { Imersão em água e gelo a } \\
13-14^{\circ} \mathrm{C} \text { e controle, ambos } \\
\text { por } 15^{\prime} \text {. }\end{array}$ & $\begin{array}{l}\text { Potência pico, trabalho total, } \\
\text { [Lac] e FC. }\end{array}$ & $\begin{array}{l}\text { A potência pico, trabalho total, [Lac] e FC foram reduzidos quan- } \\
\text { do utilizada a imersão no primeiro teste, comparado com o } \\
\text { grupo controle. }\end{array}$ \\
\hline Bailey et al. ${ }^{(17)}$ & $\begin{array}{l}20 \text { sujeitos do } \\
\text { sexo masculino } \\
\text { (22,3 } \pm 3,3 \text { anos). }\end{array}$ & $\begin{array}{l}90^{\prime} \text { de exercício intermitente } \\
\text { - corrida. }\end{array}$ & $\begin{array}{l}\text { Imersão em água e gelo a } \\
10 \pm 0,5^{\circ} \mathrm{C} \text { e controle, ambos } \\
\text { por } 15^{\prime} \text {. }\end{array}$ & $\begin{array}{l}\text { Percepção de dor, contração } \\
\text { isométrica máxima, CK e mio- } \\
\text { globina. }\end{array}$ & $\begin{array}{l}\text { A imersão reduziu o dolorimento muscular na } 1,24 \text { e } 48 \text { h após o } \\
\text { esforço quando comparado com o controle. A imersão diminuiu a } \\
\text { contração isométrica máxima e a concentração de mioglobina. }\end{array}$ \\
\hline
\end{tabular}


Além disso, as frágeis descrições dos modelos utilizados apresentam limitação importante para garantir a reprodutibilidade dos experimentos e, portanto, vários achados relacionados a esse método de recuperação mostram-se controversos. Dessa forma, entende-se que mais estudos serão necessários, para possíveis conclusões.

\section{Recuperação ativa}

Apesar de a recuperação ativa ser a técnica mais antiga, em se tratando de recuperação pós-exercício ${ }^{(3)}$, esta ainda vem sendo muito estudada.

Dupont et al. ${ }^{(19)}$ utilizaram recuperação ativa com exercício a 40\% do $\mathrm{VO}_{2 \text { máx }}$ em cicloergômetro. Diferentemente de Takahashi et al.(20), que utilizaram 20\% do $\mathrm{VO}_{2 \text { máx }}$ também em cicloergômetro por cinco minutos. Embora ambos os estudos tivessem obtido êxito, os resultados mostraram que a recuperação ativa, quando comparada com a passiva, apresenta aumento do volume sistólico e débito cardíaco(20), melhor saturação parcial de oxigênio, aumento do tempo de exaustão e potência metabólica(19).

Para Dupont et al.(21), o ideal é que o exercício ativo esteja em 50\% do $\mathrm{VO}_{2 \text { máxi }}$ este não deve ser realizado por tempo prolongado, pois, cargas de maior duração não são adequadas ao objetivo de compensação e levariam à sobrecarga no sistema cardiocirculatório, bem como gasto energético e desgaste dos tecidos ${ }^{(12)}$.

Em estudo com 20 sujeitos classificados como fisicamente ativos, Connolly et al.(25) utilizaram recuperação ativa por três minutos com 80rpm em cicloergômetro. Gill et al. (37) utilizaram semelhante protocolo, diferindo em relação ao tempo de exercício ativo e à população de estudo, formada por 23 jogadores de rúgbi de alto rendimento. Para Gill et al. ${ }^{(37)}$, os resultados demonstraram 88,2\% de recuperação, se tratando de remoção de CK. Os resultados de Connolly et al. ${ }^{(25)}$ não se apresentaram muito satisfatórios, embora a concentração de lactato tenha diminuído nos primeiros momentos de recuperação ativa, esse valor foi se igualando quando comparado com o da recuperação passiva.
Provavelmente, a principal razão para diferença de resultados esteja relacionada mais com o estado de treinamento dos indivíduos do que com a diferença nos exercícios ${ }^{(9)}$, ou seja, quanto mais alto for o nível de aptidão do sujeito, mais alta será a intensidade do exercício de recuperação para adequada remoção de ácido lático(3).

Como nas outras técnicas, na recuperação ativa, não se sabe claramente qual o tipo e a intensidade do exercício, bem como o tempo de exposição mais adequado para remoção ótima de catabólitos produzidos durante o esforço de alta intensidade. Alguns autores acreditam que essa intensidade deva estar entre 20 e 40\% do $\mathrm{VO}_{2 \text { máx }}(18-20,22,23)$, outros referem $50 \% \mathrm{VO}_{2 \text { máx }}{ }^{(11,21)}$. Apesar dessa considerável margem de 20-50\% do $\mathrm{VO}_{2 \text { máx }}$ Barnett ${ }^{(3)}$ descreve que o principal fator relacionado com a prescrição de exercício ativo é a capacidade física do sujeito, ressaltando o tempo, o tipo de exercício, bem como a intensidade do mesmo para promover eficiente recuperação.

\section{CONSIDERAÇÕES FINAIS}

Do ponto de vista metodológico, técnicas como crioterapia, contraste e massagem necessitam de maior embasamento clínico para sua aplicabilidade. Atualmente, tais modelos de recuperação têm sido usados com frequência, mas seus resultados são questionáveis devido à escassez de informações sobre os aspectos fisiológicos envolvidos no fenômeno e à falta de padronização das rotinas para coleta de informações.

Notou-se que quaisquer modelos de recuperação podem apresentar falhas em relação ao controle das variáveis e, portanto, devem ser mais bem estudados. Tanto na crioterapia como no contraste, deve-se atentar principalmente para a temperatura. Na massagem, a pressão exercida e a técnica; já na recuperação ativa, o tipo e a intensidade de esforço.

Ressalta-se que o tempo de exposição é de fundamental importância para todos os métodos. Entretanto, diversos estudos não se propõem a identificar os reais efeitos fisiológicos promovidos pelas técnicas, utilizando-as de modo insipiente. Portanto, a inconsistência

Tabela 2. Síntese das pesquisas relacionando massagem e recuperação pós-exercício

\begin{tabular}{|c|c|c|c|c|c|}
\hline Autor & $\begin{array}{l}\text { População de } \\
\text { estudo }\end{array}$ & $\begin{array}{l}\text { Modelo de estresse e nível de } \\
\text { controle }\end{array}$ & $\begin{array}{l}\text { Modelo de } \\
\text { recuperação e } \\
\text { controle }\end{array}$ & Variáveis estudadas & Resultados \\
\hline Zainuddin et al.(41) & $\begin{array}{l}10 \text { sujeitos de } \\
\text { ambos os sexos } \\
(23 \pm 1,3 \text { anos })\end{array}$ & $\begin{array}{l}60 \text { contrações máximas dos } \\
\text { músculos flexores do cotovelo, } \\
\text { divididas em } 10 \text { séries e com 3'} \\
\text { de intervalo entre as séries, num } \\
\text { dinamômetro isocinético. }\end{array}$ & $\begin{array}{l}\text { Massagem e grupo con- } \\
\text { trole, ambos por } 10^{\prime}\end{array}$ & $\begin{array}{l}\text { Força máxima isomé- } \\
\text { trica e isotônica, am- } \\
\text { plitude de movimento, } \\
\text { circunferência do mem- } \\
\text { bro, CK, dolorimento } \\
\text { muscular. }\end{array}$ & $\begin{array}{l}\text { Dor muscular tardia foi menor no grupo mas- } \\
\text { sagem. O pico de CK foi inferior ao do grupo } \\
\text { controle no } 4^{\circ} \text { dia pós-exercício. }\end{array}$ \\
\hline Mori et al. ${ }^{(42)}$ & $\begin{array}{l}29 \text { sujeitos do sexo } \\
\text { masculino }(22,4 \pm 4,2 \\
\text { anos })\end{array}$ & $\begin{array}{l}\text { Posição pronada, extensão do } \\
\text { tronco por } 90^{\prime \prime}\end{array}$ & $\begin{array}{l}\text { Massagem e grupo } \\
\text { controle, ambos por } 5 .\end{array}$ & $\begin{array}{l}\text { Fluxo sanguíneo da pele } \\
\text { e do músculo, tempera- } \\
\text { tura da pele e sensação } \\
\text { de fadiga. }\end{array}$ & $\begin{array}{l}\text { Houve aumento do fluxo sanguíneo muscu- } \\
\text { lar e da pele no grupo massagem, quando } \\
\text { comparado com o grupo controle. A esca- } \\
\text { la visual analógica apresentou menor valor } \\
\text { quando utilizada a massagem, além de haver } \\
\text { aumento da temperatura da pele para essa } \\
\text { mesma técnica. }\end{array}$ \\
\hline Brooks et al. ${ }^{(38)}$ & $\begin{array}{l}52 \text { sujeitos de } \\
\text { ambos os sexos } \\
\text { (39 } \pm 13,63 \text { anos) }\end{array}$ & $\begin{array}{l}\text { Dinamômetro isocinético por 3' } \\
\text { que produzisse índice de fadiga } \\
\text { de } 60 \% \text { da força. Membros su- } \\
\text { periores. }\end{array}$ & $\begin{array}{l}\text { Massagem e grupo } \\
\text { controle, ambos por } 5 \text {. }\end{array}$ & $\begin{array}{l}\text { Desempenho da potên- } \\
\text { cia de força. }\end{array}$ & $\begin{array}{l}\text { A massagem auxiliou num melhor desem- } \\
\text { penho de força comparado com o grupo } \\
\text { controle. }\end{array}$ \\
\hline Robertson et al. ${ }^{(40)}$ & $\begin{array}{l}9 \text { sujeitos do sexo } \\
\text { masculino entre } 20 \\
\text { e } 22 \text { anos }\end{array}$ & $\begin{array}{l}6 \text { testes num cicloergômetro cada } \\
\text { um com } 30^{\prime \prime} \text { de duração, finalizan- } \\
\text { do com o teste de Wingate. }\end{array}$ & $\begin{array}{l}\text { Massagem e grupo con- } \\
\text { trole, ambos por } 20^{\prime}\end{array}$ & $\begin{array}{l}\text { [Lac], FC, potência pico, } \\
\text { potência média e índice } \\
\text { de fadiga. }\end{array}$ & $\begin{array}{l}\text { O índice de fadiga medido quando utilizada } \\
\text { massagem foi menor quando comparado } \\
\text { com o grupo controle. }\end{array}$ \\
\hline
\end{tabular}


Tabela 3. Síntese das pesquisas relacionando exercício ativo e recuperação pós-exercício

\begin{tabular}{|c|c|c|c|c|c|}
\hline Autor & População de estudo & $\begin{array}{l}\text { Modelo de estresse e } \\
\text { nível de controle }\end{array}$ & $\begin{array}{c}\text { Modelo de recuperação } \\
\text { e controle }\end{array}$ & Variáveis estudadas & Resultados \\
\hline Connolly et al..(25) & $\begin{array}{l}7 \text { ciclistas do sexo mas- } \\
\text { culino ( } 21,8 \pm 3,3 \text { anos). }\end{array}$ & $\begin{array}{l}6 \text { séries de } 15^{\prime \prime} \text { de } 5,5 \mathrm{~kg} \text { de } \\
\text { resistência a } 80 \mathrm{rpm} \text { num ci- } \\
\text { cloergômetro com intervalo } \\
\text { de } 3^{\prime} \text { entre as séries. }\end{array}$ & $\begin{array}{l}\text { Recuperação ativa (ACT)- } \\
80 \text { rpm com } 1 \mathrm{~kg} \text { de resis- } \\
\text { tência por } 3^{\prime} \text {; e recupera- } \\
\text { ção passiva (PAS)-2' } 50^{\prime \prime} \\
\text { repouso e } 10^{\prime \prime} \text { pedalando } \\
\text { levemente. }\end{array}$ & [Lac], potência pico. & $\begin{array}{l}\text { A [Lac] esteve com valores menores durante a } \\
\text { maior parte do tempo no teste com ACT, po- } \\
\text { rém ao final do teste o valor do lactato quase } \\
\text { se igualou quando comparado com o teste de } \\
\text { PAS. A potência pico aumentou na ACT ao final } \\
\text { do sexto período de exercício. }\end{array}$ \\
\hline Dupont et al. ${ }^{(19)}$ & $\begin{array}{l}12 \text { estudantes do sexo } \\
\text { masculino que treinam } \\
\text { futebol de } 3 \text { a } 5 \text { vezes } \\
\text { por semana }(24,3 \pm 4,1 \\
\text { anos). }\end{array}$ & $\begin{array}{l}\text { Exercício intermitente em ci- } \\
\text { cloergômetro (15" de esforço } \\
\text { máximo alternado com } 15^{\prime \prime} \\
\text { de recuperação - ativa ou } \\
\text { passiva) }\end{array}$ & $\begin{array}{l}\text { PAS (repouso); e ACT } \\
\left(40 \% \text { do } \mathrm{VO}_{2 \text { máx }} \text { em ci- }\right. \\
\text { cloergômetro) }\end{array}$ & $\begin{array}{l}\text { Saturação parcial de } \mathrm{O}_{2} \\
\text { tempo de exaustão, pa- } \\
\text { râmetros cardiorrespira- } \\
\text { tórios, [Lac] e potência } \\
\text { metabólica. }\end{array}$ & $\begin{array}{l}\text { Tempo de exaustão foi menor na PAS; satu- } \\
\text { ração parcial de } \mathrm{O}_{2} \text { esteve melhor na ACT; } \\
\text { potência metabólica foi maior na ACT. }\end{array}$ \\
\hline Susuki et al. ${ }^{(7)}$ & 15 jogadores de rúgbi & Partida de rúgbi. & $\begin{array}{l}\text { Após a partida, } 7 \text { atletas } \\
\text { seguiam suas atividades } \\
\text { diárias e os outros } 8 \text { rea- } \\
\text { lizaram exercício de baixa } \\
\text { intensidade na água por } \\
1 \mathrm{~h} .\end{array}$ & $\begin{array}{l}\text { Atividade fagocitária do } \\
\text { neutrófilo e leucócito, es- } \\
\text { cala para avaliar a condi- } \\
\text { ção psicológica, glutamato } \\
\text { oxalacetato transaminase, } \\
\text { glutamato piruvato transa- } \\
\text { minase, CK e [Lac]. }\end{array}$ & $\begin{array}{l}\text { A concentração de neutrófilo e de leucócito } \\
\text { esteve aumentada após a partida em ambos } \\
\text { os grupos. Após } 1 \text { dia a concentração voltou } \\
\text { aos valores pré-jogo. A CK aumentou em am- } \\
\text { bos os grupos após a partida e continuou a } \\
\text { aumentar no dia seguinte. A variação da [Lac] } \\
\text { e da CK foi similar em ambos os grupos. }\end{array}$ \\
\hline Takahashi et al. ${ }^{(20)}$ & $\begin{array}{l}7 \text { sujeitos do sexo mas- } \\
\text { culino entre } 21-27 \\
\text { anos. }\end{array}$ & $\begin{array}{l}10^{\prime} \text { de cicloergômetro a } \\
60 \% \mathrm{VO}_{2 \text { máx. }}\end{array}$ & $\begin{array}{l}5^{\prime} \text { de PAS, posição supina; } \\
\text { e 5' de ACT a } 20 \% V_{2 \text { máx }} \\
\text { no cicloergômetro a } \\
60 \text { rpm. }\end{array}$ & $\begin{array}{l}\text { FC, volume sistólico e dé- } \\
\text { bito cardíaco. }\end{array}$ & $\begin{array}{l}\text { Na posição supina, a FC durante a ACT foi me- } \\
\text { nor que a PAS. Na posição em pé o volume } \\
\text { sistólico diminuiu durante a PAS, enquanto } \\
\text { que na ACT o nível permaneceu. O débito } \\
\text { cardíaco na posição em pé foi menor na PAS } \\
\text { quando comparado com o ACT. }\end{array}$ \\
\hline $\begin{array}{l}\text { Tardieu-Berger } \\
\text { et al. }{ }^{(26)}\end{array}$ & $\begin{array}{l}11 \text { atletas de resistência } \\
(16,6 \pm 0,4 \text { anos). }\end{array}$ & $\begin{array}{l}30 " \text { correndo a } 110 \% \text { da } \\
\text { máxima velocidade aeróbi- } \\
\text { ca alternando com } 50 \% \text { no } \\
\text { mesmo tempo, até a exaus- } \\
\text { tão (IE). Outra maneira foi } \\
\text { adicionar ACT e PAS entre } \\
\text { duas séries desse mesmo } \\
\text { protocolo supracitado (IEs). }\end{array}$ & $\begin{array}{l}\text { PAS 30", ACT } 50 \% \text { da má- } \\
\text { xima velocidade aeróbia } \\
\text { por 3' e finalmente PAS } \\
\text { 30"; entre as duas séries. }\end{array}$ & $\begin{array}{l}\text { Gases respiratórios, [Lac], } \\
\text { tempo de exaustão e o } \\
\text { tempo gasto para alcançar } \\
90 \% \mathrm{VO}_{2 \text { máx}}\end{array}$ & $\begin{array}{l}\mathrm{O} \mathrm{VO}_{2} \text { foi menor no IEs quando comparado } \\
\text { com o IE. O tempo de exaustão no IEs foi } \\
\text { maior. Entretanto quando o valor é expresso } \\
\text { em } \% \text { tanto pra } \mathrm{VO}_{2} \text { e } t 90 \% \mathrm{VO}_{2 \text { máx }} \text { O IEs torna- } \\
\text { se menor. } \mathrm{A}[\mathrm{LaC}] \text { tendeu a aumentar durante } \\
\text { cada série e diminuir ou estabilizar no período } \\
\text { de recuperação. }\end{array}$ \\
\hline Spierer et al.(23) & $\begin{array}{l}6 \text { sujeitos sedentários } \\
\text { ( } 32 \pm 1 \text { ano) e } 9 \text { fisicamen- } \\
\text { te ativos ( } 22 \pm 1 \text { ano). }\end{array}$ & Teste de Wingate. & $\begin{array}{l}\mathrm{ACT}\left(28 \% \mathrm{VO}_{2 \text { máx }}\right) \text { e PAS } \\
\text { ambos por } 4^{\prime} .\end{array}$ & $\begin{array}{l}\text { [Lac], FC, pico e media } \\
\text { de potência, índice de } \\
\text { fadiga. }\end{array}$ & $\begin{array}{l}\text { A potência média foi maior na ACT. O nível } \\
\text { de lactato foi menor na ACT para os sujeitos } \\
\text { fisicamente ativos, mas não diferiu da PAS nos } \\
\text { sujeitos sedentários. }\end{array}$ \\
\hline
\end{tabular}

Tabela 4. Síntese das pesquisas relacionando dois métodos ou mais à recuperação pós-exercício

\begin{tabular}{|c|c|c|c|c|c|}
\hline Autor & $\begin{array}{l}\text { População de } \\
\text { estudo }\end{array}$ & $\begin{array}{l}\text { Modelo de estresse e nível } \\
\text { de controle }\end{array}$ & $\begin{array}{l}\text { Modelo de recuperação } \\
\text { e controle }\end{array}$ & Variáveis estudadas & Resultados \\
\hline Gill et al. ${ }^{(37)}$ & $\begin{array}{l}23 \text { jogadores } \\
\text { de rúgbi de alto } \\
\text { rendimento }(25 \pm 3 \\
\text { anos). }\end{array}$ & Após partida de rúgbi & $\begin{array}{l}\text { ACT por } 7^{\prime} \text { com 80-100rpm, } \\
\sim 150 \text {; PAS por 9'; contraste } \\
\text { (CWT) - 1'a } 8^{\prime}-10^{\circ} \mathrm{C} \text { e } 2^{\prime} \text { a } 40-42^{\circ} \mathrm{C} \\
\text { por 9'; e um vestuário (compres- } \\
\text { são- GAR) por } 12 \text { horas. }\end{array}$ & CK & $\begin{array}{l}\text { A ACT mostrou 88,2\% de recuperação; a } \\
\text { GAR e contraste foram de } 84,4 \% \text { e } 85 \% \\
\text { respectivamente, após } 84 \mathrm{~h} \text {. A PAS foi } \\
\text { de } 39 \% \text { em relação aos outros métodos } \\
\text { tanto em } 36 \mathrm{~h} \text { quanto em } 84 \mathrm{~h} \text {. }\end{array}$ \\
\hline Coffey et al..(18) & $\begin{array}{l}14 \text { sujeitos do } \\
\text { sexo masculino } \\
\text { fisicamente ativos } \\
\text { (26,4 } \pm 6,6 \text { anos). }\end{array}$ & $\begin{array}{l}\text { Início com } 120 \% \text { da velocidade } \\
\text { máxima (Vmáx), até a exaustão, } \\
\text { seguido de recuperação por 15'(8' } \\
\text { a 40\% Vmáx, 5'sentado, } 60^{\prime \prime} \text { aqueci- } \\
\text { mento a } 40 \% \text { Vmáx, e } 60^{\prime \prime} \text { correndo } \\
\text { na esteira); após isso corrida a } 90 \% \\
\text { da Vmáx até a exaustão seguido de } \\
\text { uma técnica recuperativa. }\end{array}$ & $\begin{array}{l}\text { ACT (40\%Vmáx); PAS (repouso); } \\
\text { e CWT ao nível da EIAS (1'frio- } \\
10^{\circ} \mathrm{C} / 2^{\prime} \text { quente- } 42^{\circ} \mathrm{C} \text { ) todos } \\
\text { por } 15^{\prime} \text {. }\end{array}$ & $\begin{array}{l}\text { FC, pH, [Lac] e percep- } \\
\text { ção da recuperação. }\end{array}$ & $\begin{array}{l}\text { A [Lac] foi mais baixa na ACT e no CWT } \\
\text { quando comparado com a PAS. A FC } \\
\text { esteve aumentada na ACT entre o } 4 \text { e } \\
\text { o } 12^{\prime} \text { comparado com a PAS e entre o } \\
8 \text { e } 12^{\prime} \text { comparado com o CWT. Os vo- } \\
\text { luntários relataram melhor percepção } \\
\text { na recuperação por CWT. }\end{array}$ \\
\hline Hart et al. ${ }^{(10)}$ & $\begin{array}{l}19 \text { sujeitos de } \\
\text { ambos os sexos } \\
(20,6 \pm 1,2 \text { ano })\end{array}$ & $\begin{array}{l}4 \text { ou } 5 \text { séries de } 35 \text { repetições de } \\
\text { flexão plantar do tornozelo com } \\
90 \% \text { de } 1 \text { RM. }\end{array}$ & $\begin{array}{l}\text { ACT - resistência constante a } \\
90 \% \text { s e massagem, ambos por } 5^{\prime} \text {. }\end{array}$ & $\begin{array}{l}\text { Circunferência do } \\
\text { membro e escala visu- } \\
\text { al analógica de dor. }\end{array}$ & $\begin{array}{l}\text { Não houve significância entre as variá- } \\
\text { veis estudadas. }\end{array}$ \\
\hline Monedero et al. ${ }^{(11)}$ & $\begin{array}{l}18 \text { ciclistas do sexo } \\
\text { masculino. }\end{array}$ & $\begin{array}{l}\text { Dois momentos de } 5 \mathrm{~km} \text { num } \\
\text { cicloergômetro, intensidade indi- } \\
\text { vidualizada. }\end{array}$ & $\begin{array}{l}\text { ACT }\left(50 \% V_{2} O_{2 a ́ x}\right) ; \text { PAS; massa- } \\
\text { gem; ACT e massagem. Todas } \\
\text { por 15'. }\end{array}$ & [Lac], FC. & $\begin{array}{l}\text { ACT e massagem combinadas foram me- } \\
\text { Ihores que os outros métodos para man- } \\
\text { ter o desempenho no segundo teste. A } \\
\text { ACT foi melhor na remoção da [LaC]. }\end{array}$ \\
\hline Lane et al.(22) & $\begin{array}{l}10 \text { sujeitos do sexo } \\
\text { masculino }(26,3 \pm 2 \\
\text { anos) }\end{array}$ & $\begin{array}{l}\text { 18' de exercício intermitente num } \\
\text { cicloergômetro separado por } 24 \\
\text { horas. }\end{array}$ & $\begin{array}{l}\mathrm{ACT}\left(30 \% \mathrm{VO}_{2 \text { máx }}\right) \text {, massagem, } \\
\text { imersão em água e gelo a } 15^{\circ} \mathrm{C} \\
\text { e controle. Todas por } 15^{\prime} \text {. }\end{array}$ & $\begin{array}{l}\text { Comparar o desem- } \\
\text { penho entre o } 1^{\circ} \mathrm{e} \mathrm{o} \\
2^{\circ} \text { teste. }\end{array}$ & $\begin{array}{l}\text { O grupo controle teve desempenho } \\
\text { inferior quando comparado com os } \\
\text { demais métodos. }\end{array}$ \\
\hline
\end{tabular}


dos resultados encontrados sugere que as variáveis utilizadas como método de recuperação devem ser melhor controladas.

Ainda no âmbito do controle das variáveis, muitos estudos, examinando a eficácia das técnicas recuperativas, têm-se focado preferencialmente nas análises de lactato e $\mathrm{CK}^{(8,17,27,36)}$. Embora cada técnica recuperativa demonstre efetividade em alguns estudos, outras variáveis poderiam ser melhor estudadas, como CK na isoforma MM, conforme sugere Brancaccio et al.(43), proteína C-reativa ${ }^{(44)}$ e interleucina-1(45), que repercutem danos teciduais, além da variabilidade de frequência cardíaca, sendo esse um instrumento de metodologia simples e não-invasiva e que demonstra a atividade autonômica do coração(46).

Especificamente sobre as técnicas, as que parecem ter efeitos semeIhantes são o contraste e a recuperação ativa, no que tange à remoção de lactato e diminuição da CK. Estudos mostram que não há diferença significante quando comparados os dois métodos, embora haja melhor percepção de recuperação quando utilizado o contraste ${ }^{(18,37)}$.

\section{REFERÊNCIAS BIBLIOGRÁFICAS}

1. Sellwood KL, Brukner P, Williams D, Nicol A, Hinman R. Ice-water immersion and delayed-onset muscle soreness: a randomised controlled trial. Br J Sports Med. 2007;41(6):392-7.

2. Wilcock IM, Cronim JB, Hing WA. Physiological response to water immersion: a method for sport recovery? Sports Med. 2006;36(9):747-65. Review.

3. Barnett A. Using recovery modalities between training sessions in elite athletes: does it help? Sports Med. 2006;36(9):781-96. Review.

4. Tomlin DL, Wenger HA. The relationship between aerobic fitness and recovery from high intensity intermittent exercise. Sports Med. 2001;31(1):1-11.

5. Burke LM, Kiens B, Ivy JL. Carbohydrates and fat for training and recovery. J Sports Sci. 2004;22(1):1530. Review.

6. Ohnishi N, Yamane M, Uchiyama N, Shirasawa S, Kosaka M, Shiono H, et al. Adaptive changes in muscular performance and circulation by resistance training with regular cold application. J Therm Biol. 2004;29:839-43.

7. Suzuki M, Umeda T, Nakaji S, Shimoyama T, Mashiko T, Sugawara K. Effect of incorporating low intensity exercise into the recovery period after a rugby match. Br J Sports Med. 2004;38(4):436-40.

8. Yanagisawa O, Niitsu M, Yoshioka H, Goto K, Kudo H, Itai Y. The use of magnetic resonance imaging to evaluate the effects of cooling on skeletal muscle after strenuous exercise. Eur J Appl Physiol. 2003;89(1):53-62.

9. Cheung K, Hume P, Maxwell L. Delayed onset muscle soreness: treatment strategies and performance factors. Sports Med. 2003;33(2):145-64. Review.

10. Hart JM, Swanik CB, Tierney RT. Effects of sport massage on limb girth and discomfort associated with eccentric exercise. J Athl Train. 2005;40(3):181-5.

11. Monedero J, Donne B. Effect of recovery interventions on lactate removal and subsequent performance. Int J Sports Med. 2000;21(8):593-7.

12. Sairyo K, Iwanaga K, Yoshida N, Mishiro T, Terai T, Sasa T, et al. Effects of active recovery under a decreasing work load following intense muscular exercise on intramuscular energy metabolism. Int J Sports Med. 2003;24(3):179-82.

13. Cochrane DJ. Alternating hot and cold water immersion for athlete recovery: a review. Phys Ther Sports. 2004;5:26-32.

14. Morton RH. Contrast water immersion hastens plasma lactate decrease after intense anaerobic exercise. J Sci Med Sport. 2007;10(6):467-70.

15. Paddon-Jones DJ, Quigley BM. Effect of cryotherapy on muscle soreness and strength following eccentric exercise. Int J Sports Med. 1997;18(8):588-93

16. Eston R, Peters D. Effects of cold water immersion on the symptoms of exercise-induced muscle damage. J Sports Sci. 1999;17(3):231-8.

17. Bailey DM, Erith SJ, Griffin PJ, Dowson A, Brewer DS, Gant N, et al. Influence of cold-water immersion on indices of muscle damage following prolonged intermittent shuttle running. J Sports Sci. 2007;25(11):1163-70.

18. Coffey V, Leveritt M, Gill N. Effect of recovery modality on 4-hour repeated treadmill running performance and changes in physiological variables. J Sci Med Sport. 2004;7(1):1-10.

19. Dupont G, Moalla W, Guinhouya C, Ahmaidi S, Berthoin S. Passive versus active recovery during highintensity intermittent exercises. Med Sci Sports Exerc. 2004;36(2):302-8.

20. Takahashi T, Hayano J, Okada A, Saitoh T, Kamiya A. Effects of the muscle pump and body posture on cardiovascular responses during recovery from cycle exercise. Eur J Appl Physiol. 2005;94(5-6):576-83.

21. Dupont G, Blondel N, Berthoin S. Performance for short intermittent runs: active recovery vs. passive recovery. Eur J Appl Physiol. 2003;89(6):548-54

22. Lane KN, Wenger HA. Effect of selected recovery conditions on performance of repeated bouts of intermittent cycling separated by 24 hours. J Strength Cond Res. 2004;18(4):855-60.

23. Spierer DK, Goldsmith R, Baran DA, Hryniewicz K, Katz SD. Effects of active vs. passive recovery on work performed during serial supramaximal exercise tests. Int J Sports Med. 2004;25(2):109-14.
Na comparação entre exercício físico e massagem, observa-se que o primeiro parece ser mais eficiente para remoção de lactato, todavia, a combinação destes métodos mostra-se adequada para manter o desempenho máximo em exercício(11).

Por fim, entende-se que os vieses descritos e a complexidade nos processos de comparação entre as diferentes técnicas, sobretudo pela incongruência nos modelos de controle de variáveis, podem estar relacionados com a distância existente entre as práticas de campo e pesquisas em laboratório. Dessa forma, com este estudo de revisão sistemática, a partir dos resultados descritos por outrem e hipóteses levantadas para explicar tais eventos, pretendeu-se contribuir, como elemento facilitador, visando nortear os cientistas do esporte a empreender investigações pertinentes à realidade do campo esportivo para o tema em questão.

$\overline{\text { Todos os autores declararam não haver qualquer potencial conflito de }}$ interesses referente a este artigo.
24. Weerapong P, Hume PA, Kolt GS. The mechanisms of massage and effects on performance, muscle recovery and injury prevention. Sports Med. 2005;35(3):235-56.

25. Connolly DAJ, Brennan KM, Lauzon CD. Effects of active versus passive recovery on power output during repeated bouts of short term, high intensity exercise. J Sports Sci Med. 2003;2:47-51.

26. Tardieu-Berger M, Thevenet D, Zouhal H, Prioux J. Effects of active recovery between series on performance during an intermittent exercise model in young endurance athletes. Eur J Appl Physiol. 2004:93(1-2):145-52.

27. Crowe MJ, O'Connor D, Rudd D. Cold water recovery reduces anaerobic performance. Int J Sports Med. 2007;28(12):994-8.

28. Armstrong LE, Crago AE, Adams R, Roberts WO, Maresh CM. Wole-body cooling of hyperthermic runners: comparison of two field therapies. Am J Emerg Med. 1996;14(4):355-8.

29. Yeargin SW, Casa DJ, McClung JM, Knight JC, Healey JC, Goss PJ, et al. Body cooling between two bouts of exercise in the heat enhances subsequent performance. J Strength Cond Res. 2006;20(2):383-9.

30. Douris $P$, McKenna R, Madigan K, Cesarski B, Costiera R, Lu M. Recovery of maximal isometric grip strength following cold immersion. J Strength Cond Res. 2003;17(3):509-13.

31. Isabell WK, Durrant E, Myrer W, Anderson S. The Effects of Ice Massage, Ice Massage With Exercise, and Exercise on the Prevention and Treatment of Delayed Onset Muscle Soreness. J Athl Train. 1992;27(3):208-17.

32. Yackzan L, Adams C, Francis KT. The effects of ice massage on delayed muscle soreness. Am J Sports Med. 1984;12(2):159-65.

33. Howatson G, Van Someren KA. Ice massage. Effects on exercise-induced muscle damage. J Sports Med Phys Fitness. 2003;43(4):500-5.

34. Pöyhönen T, Avela J. Effect of head-out water immersion on neuromuscular function of the plantarflexor muscles. Aviat Space Environ Med. 2002;73(12):1215-8.

35. Hornery DJ, Papalia S, Mujika I, Hahn A. Physiological and performance benefits of halftime cooling. J Sci Med Sport. 2005;8(1):15-25.

36. Howatson G, Gaze D, van Someren KA. The efficacy of ice massage in the treatment of exercise-induced muscle damage. Scand J Med Sci Sports. 2005;15(6):416-22.

37. Gill ND, Beaven CM, Cook C. Effectiveness of post-match recovery strategies in rugby players. $\mathrm{Br} J$ Sports Med. 2006;40(3):260-3.

38. Brooks CP, Woodruff LD, Wright LL, Donatelli R. The immediate effects of manual massage on power-grip performance after maximal exercise in healthy adults. J Altern Complement Med. 2005;11(6):1093-101

39. Moraska A. Sports massage. A comprehensive review. J Sports Med Phys Fitness. 2005;45(3):370-80.

40. Robertson A, Watt JM, Galloway SD. Effects of leg massage on recovery from high intensity cycling exercise. Br J Sports Med. 2004;38(2):173-6.

41. Zainuddin Z, Newton M, Sacco P, Nosaka K. Effects of massage on delayed-onset muscle soreness, swelling, and recovery of muscle function. J Athl Train. 2005;40(3):174-80.

42. Mori H, Ohsawa H, Tanaka TH, Taniwaki E, Leisman G, Nishijo K. Effect of massage on blood flow and muscle fatigue following isometric lumbar exercise. Med Sci Monit. 2004;10(5):CR173-8.

43. Brancaccio P, Maffulli N, Limongelli FM. Creatine kinase monitoring in sport medicine. Br Med Bull. 2007;81-82:209-30.

44. Peters EM, Robson PJ, Kleinveltdt NC, Naicker VL, Jogessar VD. Hematological recovery in male ultramarathon runners: the effect of variations in training load and running time. J Sports Med Phys Fitness. 2004;44(3):315-21

45. Smith LL. Acute inflammation: the underlying mechanism in delayed onset muscle soreness? Med Sci Sports Exerc. 1991;23(5):542-51. Review.

46. Cottin F, Médigue C, Lopes P, Leprêtre PM, Heubert R, Billat V. Ventilatory thresholds assessment from heart rate variability during an incremental exhaustive running test. Int J Sports Med. 2007;28(4):287-94. 